\title{
De la « pause » à la « valeur » en langue : grammaticalisation des signes de ponctuation?
}

\author{
Rault Julien \\ Université de Poitiers \\ julien.rault01@univ-poitiers.fr
}

Le prisme de l'oral et de la voix a longtemps été considéré comme le critère discriminant dans l'approche de la ponctuation. Ainsi, le point de vue phonocentriste plaçait la question de la respiration au cœur des enjeux et faisait des signes de ponctuation des marqueurs prosodiques en lien avec le souffle et le corps ${ }^{1}$. Il est évident que l'encodage de l'oralisation ne suffit pas à rendre compte du fonctionnement de la ponctuation; l'oral ne peut être, comme le dit Anne-Marie Christin à propos de l'écriture, « la référence ultime et exclusive de l'écrit $»^{2}$.

Sur un plan diachronique, l'examen des discours des linguistes et grammairiens montre que,

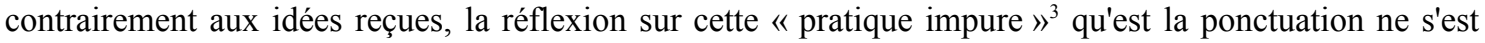
pas toujours cantonnée à l'approche prosodique. Depuis les premiers traités, jusqu'aux courants phonograhique (Catach, Védénina) et autonomiste (Anis) est perceptible un infléchissement constant vers une forme d'encodage grammatical. Ce parcours nous amène alors à poser l'hypothèse suivante : il existe un mouvement d'inclusion progressive des signes de ponctuation dans le système de la langue. Dans une telle dynamique, la proposition de Jacqueline Authier-Revuz (1995) d'établir, pour chaque signe de ponctuation, un signifié ou une "valeur en langue» pourrait constituer un moment décisif dans l'évolution des modes d'appréhension. Des travaux de recherche plus récents encore - Lefebvre sur la note de bas de page (2004), Pétillon (2002) et Serça (2004) sur les parenthèses et tirets - ont pu proposer des analyses linguistiques des signes de ponctuation qui tendent vers la mise à jour d'une valeur globale du signe. Envisager le signe de ponctuation (noire) ${ }^{4}$ en tant que signe plein, c'est-à-dire comme un ponctème, doté d'un signifiant (graphique) et d'un signifié, permettrait alors de s'interroger véritablement sur sa nature sémiotique ${ }^{5}$ et sur sa place et sa fonction tant dans le système de la langue que dans les réalisations discursives ( $«$ langue en emploi et en action $»^{6}$ ).

Nous souhaitons sonder ici plusieurs aspects relatifs à l'évolution du discours sur la ponctuation, d'un point de vue fonctionnel d'abord, en lien avec les différents rôles attribués aux éléments ponctuants (respiratoires, pausaux, syntaxiques), puis sémiotique, en interrogeant les notions de signe et de valeur. Cette étude de la morphogénèse ${ }^{7}$ de la ponctuation nous conduira à investir la notion de syntaxicalisation et de grammaticalisation. Est-il possible d'intégrer l'évolution des signes de ponctuation dans un processus plus global de transformations linguistiques?

\section{Syntaxicalisation}

Une approche détaillée des discours sur la ponctuation met en évidence le fait que les critères syntaxique et sémantique n'ont jamais été complètement ignorés des commentateurs. Nous nous proposons d'effectuer, dans un premier temps, une synthèse diachronique mettant en avant quelques descriptions particulièrement éloquentes.

A partir du XVII ${ }^{\mathrm{e}}$ siècle, le système élaboré par les grammairiens est fondé sur des éléments grammaticaux : de nombreux commentateurs pointent alors la contradiction qui existe entre l'emploi du terme " pause »-perçu comme une notion orale - par ces mêmes grammairiens et les systèmes mis en place. Selon nous, ces contradictions peuvent être résolues en interrogeant véritablement le spectre sémantique du mot « pause »; ce dernier semble beaucoup plus large qu'on veut bien le croire, pouvant intégrer des notions de segmentation syntaxique et de coupure sémantique. 
Enfin, il convient de s'arrêter un instant sur cet événement déterminant, pour la syntaxicalisation de la ponctuation, que représentent l'émergence et la grammaticalisation de la notion de phrase (Seguin).

\subsection{Respiration et structuration}

Dès l'Antiquité, la ponctuation utilisée par les grammairiens et les philologues est à la fois sonore (les pneumata), permettant d'indiquer les accentuations ou plus largement la prononciation, et visuelle (« signes de coupure, de note, de rajout, de mise à la marge, de suppression et de correction, pour bien séparer le texte lui-même des gloses $»^{8}$ ).

Les grammairiens latins de l'Antiquité tardive étaient sensibles, à la suite de Denys le Thrace, à cette double fonction des signes de ponctuation. Le grammairien Dositheus donne ainsi une définition en deux versants de la ponctuation: le premier évoque la prise en compte des impératifs pneumatiques de la lecture à haute voix en définissant la ponctuation comme «l'indication d'une pause permettant la reprise de l'haleine dans le lecture »; le second met l'accent sur la distinction logique des groupes de sens en proposant la définition suivante : «l'indication d'une pause qui signale le sens achevé »?

$\mathrm{Au} \mathrm{VII}^{\mathrm{e}}$ siècle, Isidore de Séville, dans son Etymologia, propose une définition qui met principalement l'accent sur la distinction sémantique et évacue complètement la dimension pneumatique : "C'est une figure particulière placée à la manière d'une lettre, pour démontrer chaque division des mots, des sens et des vers » (Est figura propria in litterae modum posita ad demonstrandam unamquamque verbi sententiarumque ac versuum rationem $)^{10}$.

Une telle définition, si elle reste assez rare, montre que l'appréhension des signes de ponctuation pouvait s'affranchir radicalement du rapport à l'oralité pour s'orienter sur un plan syntaxique et sémantique. Il faut donc reconsidérer l'idée selon laquelle la ponctuation, dès les premiers temps, ne possédait pas de valeur syntaxique et était dotée uniquement d'un rôle « plus esthétique que grammatical $»^{11}$. D'une part, la grande variation qui peut exister entre les différentes copies d'un même texte ne permet pas d'avoir un point de vue global et cohérent, ce qu'a bien montré le travail de Christiane Marchello-Nizia sur les manuscrits médiévaux ${ }^{12}$. D'autre part, les signes diacritiques, dont le but premier est de faciliter la lecture à haute voix, correspondent à des « unités de lecture, en tous les sens du mot lecture $»^{13}:$ le geste ponctuant est donc un "acte de lecture », ultime étape d'une création partagée, avant la mise en voix ; pour Nina Catach, du $\mathrm{VI}^{\mathrm{e}}$ siècle au $\mathrm{XV}^{\mathrm{e}}$ siècle, la ponctuation est avant tout considérée comme une aide à la 《lisibilité $»^{14}$. Ce critère de lisibilité permet d'embrasser le versant de l'oralisation (lectores, legentes) et celui de l'expression du sens (liaison). La disposition des pauses doit pouvoir assurer à la fois la reprise du souffle du lecteur mais aussi l'interprétation du texte lu - les anciens ayant « parfaitement conscience que la ponctuation participe de l'interprétation des textes, voire de leur exégèse : ponctuer, c'est commenter $»^{15}-$, ce qui amène à prendre en considération l'apport sémantique des signes ponctuants, lesquels « distinguent et révèlent aux auditeurs le sens du discours »"

Aux XVII ${ }^{e}$ et XVIII ${ }^{e}$ siècles, la dimension pneumatique, attentive au souffle, à l'haleine du locuteur, est de plus en plus fréquemment complétée, de façon explicite, par une dimension grammaticale. Antoine de Furetière avançait l'idée d' « une observation grammaticale des lieux d'un discours où on doit faire de différentes pauses $»^{17}$ et Nicolas Beauzée celle d'une distribution « des sens partiels » (logique) et d'une « différence des degrés de subordination » (syntaxe) :

Le choix des ponctuations dépend de la proportion qu'il convient d'établir dans les pauses ; et cette proportion dépend de trois principes fondamentaux. $1^{\circ}$ le besoin de respirer ; $2^{\circ}$ la distinction des sens partiels qui constituent le discours ; $3^{\circ}$ la différence des degrés de subordination qui conviennent à chacun de ces sens partiels dans l'ensemble du discours. ${ }^{18}$

La conception prosodique s'enrichit plus nettement d'une approche attentive à l'organisation du sens au sein de la hiérarchie syntaxique. Le chapitre consacré à la ponctuation dans la Grammaire générale de Nicolas Beauzée est inclus dans le Livre III, "Éléments de syntaxe ». Ponctuation et syntaxe sont ainsi explicitement liées. Les trois principes évoqués dans la définition exposent les trois grandes approches qui 
domineront l'analyse de la ponctuation : respiratoire, sémantique, syntaxique. La notion de « degrés de subordination » induit une hiérarchie entre les signes, du point jusqu'à la virgule, en passant par les signes médians que sont le point-virgule et les deux-points.

\subsection{Pause}

Plus le critère syntaxique est invoqué, plus les commentateurs soulignent une forme de contradiction dans les propos des grammairiens et linguistes de l'époque. On note ainsi, non sans perplexité, la discordance née de la persistance du critère « pausal » dans l'esprit des grammairiens et la nature du système qu'ils élaborent, fondé sur des critères en réalité beaucoup plus proches de la grammaire et de la logique : le terme pause, toujours très employé, entrerait en contradiction avec les systèmes décrits ou élaborés.

Paradoxalement, les grammairiens ont continué à croire à la nature 'pausale' ou respiratoire de la ponctuation, alors qu'en fait leur système était déjà presque exclusivement fondé sur des principes grammaticaux et logiques ${ }^{19}$.

Il peut paraître alors pertinent de s'interroger sur le véritable spectre sémantique du terme « pause ». Si les grammairiens maintiennent le critère pausal dans leur définition mais élaborent dans le même temps un système fondé sur la syntaxe et la logique, c'est peut-être que le terme «pause » est, en définitive, beaucoup plus polyvalent qu'on a bien voulu le croire.

Le point de vue de Barko, entre autres, n'est tenable que dans la mesure où l'on adopte une conception restreinte du sémantisme de «pause » (exclusivement prosodique), en évacuant le lien qu'il pourrait traduire avec l'articulation logique. Nous formulons ici l'hypothèse d'un spectre sémantique beaucoup plus large du terme $\mathrm{e}^{20}$, qui permettrait de faire disparaître les contradictions problématiques exhibées par les commentateurs.

Les définitions de Furetière et Beauzée (cf. supra) sont, à ce titre, éloquentes. Dans la définition d'Antoine de Furetière, le terme «pause» est immédiatement lié à la notion d' «observation grammaticale », ce qui oriente le point de vue du côté de la structuration logique. La répartition des «pauses» est, de façon explicite ici, perçue comme la conséquence d'une réflexion fondée sur la grammaire. Le terme semble bien impliquer un point de vue syntaxique et logique. Et il est possible de supposer que son acception était polyvalente au $\mathrm{XVI}^{\mathrm{e}}, \mathrm{XVII}^{\mathrm{e}}$ et $\mathrm{XVIII}{ }^{\mathrm{e}}$ siècles; que, dès le départ, l'emploi du mot mettait autant en jeu la diction que la distinction, autant la prosodie que la syntaxe et la sémantique. Beauzée, de son côté, relie la « pause » non pas à «l'observation » (grammaticale) mais aux « proportions ». La proportion des pauses, dit-il, dépend de la respiration, de la distinction du sens et des degrés de subordination. Le mot semble bien embrasser un ensemble plus vaste que la seule prosodie, ensemble dans lequel peuvent se loger plusieurs traits - syntaxiques, sémantiques - qui accompagnent la considération pneumatique ; user du terme «pause » permet, sans doute, de tenir compte du souffle, du sens, de la syntaxe.

Si la notion de "pause» est polysémique, on comprend mieux pourquoi la Grammaire françoise simplifiée de François-Urbain Domergue (1778) n'emploie pas le terme, lui préférant celui plus largement monosémique (physiologique) de $\left\langle\right.$ repos $»^{21}$. Pour Domergue, la règle générale de la ponctuation est, dans un premier temps, liée aux différents degrés de « suspension du sens » : l'emploi du point correspond à un sens complet, celui de la virgule à une légère suspension du sens, celui du point-virgule à une suspension un peu plus forte. Mais vient ensuite, dans un second temps, la question auditive du « repos » (par la virgule notamment). Cet emploi singulier du mot « repos » dans une définition qui fait cohabiter le sémantique («suspension du sens ») et le prosodique (« repos ») pourrait valider l'idée d'une polyvalence de la « pause » (sens + repos). Le terme englobant de « pause » n'est pas utilisé car Domergue tient à maintenir dans son approche une distinction entre les deux versants.

Marie-Luce Demonet évoque également le problème lié à l'emploi de pause, cette « notion orale » qui ne tient pas compte de la pluralité des niveaux d'analyse : 


\begin{abstract}
La seule notion « orale » importante, mentionnée dans les textes théoriques est celle de pause - et non d'intonation. Or ces signes ne marquent pas seulement les pauses puisqu'ils sont avant tout une aide à la "distinctio » des parties du discours, ce que le lecteur devra oraliser quand il prononcera un texte déjà écrit, faisant naître ainsi un double sonore du texte, non plus avant mais après lui, objet de codification grammaticale et théorique. En effet, les essais de normation de la ponctuation au $\mathrm{XVI}^{\mathrm{e}}$ siècle relèvent d'abord d'une conception mentale de la langue, essentiellement sémantico-syntaxique. ${ }^{22}$
\end{abstract}

Si le terme «pause » est préféré à celui d' « intonation», c'est peut-être justement parce que le premier n'est pas uniquement «une notion orale». Le mot "pause» utilisé dans les textes théoriques n'impliquerait pas un rapport aussi étroit à l'oralité que celui d'« intonation » et permettrait davantage de refléter l'ensemble des processus mis en œuvre par les signes de ponctuation, lesquels distinguent les parties du discours selon des critères qui tendent à devenir de plus en plus sémantiques et syntaxiques. En considérant que le terme, pris dans un certain nombre d'énoncés définitionnels, embrasse des perspectives prosodiques mais aussi logiques, syntaxiques et sémantiques, on peut être amené à réenvisager les grandes approches et à dépasser la contradiction signalée par les analystes.

La polyvalence du mot «pause » est d'autant plus probante dans le contexte du XVIII ${ }^{\mathrm{e}}$ siècle où apparaît la notion de phrase, phénomène déterminant dans la syntaxicalisation, voire dans la grammaticalisation, des ponctèmes.

\title{
1.3 Phrase
}

Jusqu'à présent l'unité discursive qui servait de cadre théorique était en effet la période. Cette unité entre en concurrence, au XVIII ${ }^{\mathrm{e}}$ siècle, avec la notion émergente de « phrase » :

\begin{abstract}
Une nouvelle contrainte s'est appliquée à un domaine qu'elle avait épargné même pendant le grand siècle : le souci de l'ordre a envahi la grammaire, non plus seulement comme option métaphysique sur les rapports de la pensée et du langage (Port Royal) mais comme préoccupation de faire marcher cette représentation de la langue (dans un imaginaire nommé syntaxe), selon des lois de hiérarchie et de positivité à prétention de science exacte. Tout groupe de mots doit trouver sa place dans un processus d'analyses par niveaux. La rhétorique ayant déjà ses périodes, ses membres et les mots qui les composent, la grammaire doit aller plus loin et feindre de représenter mathématiquement la combinaison de ses mots dans des entités à la fois objectives et théoriques : on se rassurera donc en articulant les mots en propositions (en attendant de donner ses lettres de noblesse au syntagme), et les propositions en phrases, comme s'il s'agissait de choses. ${ }^{23}$
\end{abstract}

Un tel changement va entrainer une modification nette dans la façon de concevoir la ponctuation. «Fixation de la ponctuation » et "conduite maîtrisée de la phrase » constituent deux des principaux phénomènes saillants du XVIII ${ }^{e}$ siècle $^{24}$. L'idée d'une structuration logique, dont la réflexion sur le point constitue un bel exemple ${ }^{25}$, indique bien l'impossibilité d'envisager la ponctuation sur un plan uniquement prosodique. Le point de vue des discours métalinguistiques devient nettement grammatical, reléguant au second plan la dimension respiratoire qui était étroitement liée à la période, domaine de l'art oratoire. La notion de phrase supplante ainsi celle de la période et constitue un point de bascule déterminant dans le rapport hiérarchique entre approche pneumatique et approche grammaticale.

L'évolution de la phrase et des signes de ponctuation, en tout premier lieu du point, est très certainement le fruit d'une interaction : la " grammaticalisation tardive de la notion de phrase $»^{26}$, inaugurée notamment par une réflexion nouvelle sur le rôle clôturant du point, a pu à son tour influencer la perception des signes de ponctuation. Et si cette approche nouvelle relève bien, comme le précise à de nombreuses reprises Jean-Pierre Seguin, d'un sentiment linguistique, elle offre alors un argument convaincant à l'idée d'une grammaticalisation des signes de ponctuation. 


\section{Ponctème}

Avec la notion de phrase et la mise au premier plan des critères syntaxiques, le signe de ponctuation se rapproche indéniablement du système de la langue. De là l'émergence d'un questionnement sur la nature de cet élément (un signe ?) et sur la possibilité d'attribution d'une valeur.

Les travaux de Jacqueline Authier-Revuz, qui invitent à établir une valeur - en langue - des signes de ponctuation, constituent une parfaite illustration de l'infléchissement grammatical, dans la mesure où la formulation d'un signifié suppose bien une nouvelle forme de codage et d'inscription dans le domaine de la langue.

L'évolution des modes d'appréhension du signe de ponctuation, de la respiration au signifié en langue, ne peut que rappeler le processus d'évolution linguistique dit de «grammaticalisation ». Sous forme de réflexion ouverte, nous proposons d'interroger in fine l'éventuelle intégration des éléments ponctuants dans ce processus général.

\subsection{Signes}

Des « soupirs $»^{27}$ aux «ponctèmes », le cheminement de la terminologie reflète le cheminement linguistique. Ainsi le débat sur l'emploi du mot « signe» a pu constituer un tournant majeur dans leur appréhension :

Il a fallu du temps pour que le terme « signes » s'impose et qu'il désigne ce que l'on a appelé d'abord points, puis caractères, marques ou notes. C'est au XIX ${ }^{\mathrm{e}}$ siècle que l'usage s'en est généralisé [...]. ${ }^{28}$

Points, accens, distinctions ou encore marques et notae sont les ancêtres du signe de ponctuation. L'affirmation progressive du mot «signe », au sens sémiotique (signifiant et signifié), autour duquel gravite la question de la langue, va alors provoquer une réflexion déterminante.

Se pose tout d'abord la question du signifiant graphique, lequel apparaît en premier lieu comme un oxymoron fâcheux si l'on s'en tient à la lecture la plus répandue du Cours de linguistique générale qui veut que, l'écriture étant seconde par rapport à la manifestation orale, et donc étrangère, le signifiant soit exclusivement sonore. Toutefois, Michel Arrivé a pu montrer qu'en certains points de son cours, dans les sources manuscrites notamment, Ferdinand de Saussure remettait en cause la nécessaire prononciation de la langue par l'organe vocal, concluant que les mots pouvaient être transposés dans l'écriture ${ }^{29}$. L'écriture accède ainsi au statut de système de signes, ce qui permet d'envisager la transposition du signifiant acoustique en signifiant graphique puisque, comme le dit Saussure lui-même, « le signifiant linguistique n'est aucunement phonique : il est incorporel $»^{30}$.

La question du signifié peut être aussi l'objet de quelques réticences. Pour Nina Catach, les éléments de la ponctuation sont des signes « idéographiques, c'est-à-dire porteurs de sens »" des sons. Ils s'inscrivent dans la notion d'écriture plérémique, fondée « sur des signes-morphèmes ou des signes-mots $»^{32}$ (autrement dit des signifiants renvoyant à un signifié, par opposition aux écritures cénémiques, qui utilisent des «signes-syllabes » ou des «signes-phonèmes », signifiant de signifiant). Claude Tournier se situe dans le sillage de Nina Catach lorsqu'il définit la ponctuation comme « l'ensemble des graphèmes purement plérémiques, non-décomposables en unités de rang inférieur et à caractère discret $»^{33}$. Si le signe de ponctuation est comparable au $\operatorname{mot}^{34}$, il reste en revanche «nondécomposable ». Claude Tournier, qui utilise également le terme $"$ idéographique ${ }^{35}$, justifie à plusieurs reprises l'emploi du mot « signe », au sens linguistique :

\footnotetext{
Si je parle de 'signe' de ponctuation, c'est, d'une part, pour respecter la tradition, mais aussi parce qu'il s'agit bien d'un 'signe' au sens où on l'entend en linguistique, avec ses deux faces, signifiant et signifié..$^{36}$

Ce n'est pas seulement par respect pour la tradition que nous parlons de signe de ponctuation. Comme le signe linguistique, celui-ci est, en effet, constitué d'un signifiant (le ponctuant), et d'un signifié (la ponctuance), et l'expérience nous montre
} 
que le même signifiant (la majuscule, par exemple) peut avoir plusieurs signifiés

(début de phrase, nom propre, mise en valeur, etc.). ${ }^{37}$

À l'inverse, Jean-Gérard Lapacherie et Jacques Anis refusent ou l'idée de signifié ou l'idée de sens. Pour le premier, le signe de ponctuation n'est pas un signe véritable puisqu'il n'est guère possible d'établir un signifié stable, les marques de ponctuation étant largement soumises à la variété des usages ${ }^{38}$. Pour le second, on ne peut parler de «sens » dans la mesure où l'emploi autonome reste marginal ${ }^{39}$; cependant, s'il affirme qu' « attribuer un sens au sens fort du terme au ponctème est sans doute inadéquat $»^{40}$, il ne lui refuse pas pour autant un sémantisme. Pour le linguiste, les éléments ponctuo-typographiques ne constituent pas des unités significatives au même titre que les morphèmes - notamment parce qu'ils ne sont pas autonomes mais «auxiliaires», « indicateurs de la structure des énoncés et de leur statut énonciatif», facilitant l'encodage et le décodage de l'information ${ }^{41}$ - mais ils ont une valeur sémantique, ce qui les distingue des graphèmes alphabétiques. Toutefois, on peut avancer que pour un certain nombre d'entre eux («! », «?», « ...»), l'emploi autonome est tout à fait fréquent.

Le signe de ponctuation serait donc entre le graphème et le morphème. Ce qui expliquerait en outre le caractère souvent apophatique des définitions: «non-alphabétique» (ou «non-littéral»), «sans correspondant phonémique », «non-décomposable ». Comme le fait remarquer Jean Perrot :

S'il est particulièrement gênant d'en rester à une définition négative de la ponctuation, c'est que, comme l'a fait $\mathrm{C}$. Tournier, on est amené à reconnaître aux signes de ponctuation une autre propriété : 'ils ont une signification', à l'inverse des graphèmes. $^{42}$

Josette Rey-Debove s'interroge également sur la nature sémiotique des signes de ponctuation et décide de les considérer comme des «signes réels » (composés d'un signifiant et d'un signifié) de la chaîne écrite « dont le signifiant est graphique sans être littéral » ${ }^{43}$. Mais on ne peut toutefois les considérer comme des signes ordinaires. Certains seraient en effet neutres, telle la virgule qui «ne signifie rien », mais ce «"rien" est positif dans la phrase où il décide de l'interprétation sémantique, en séparant ce qui pourrait être lié $»^{44}$. D'autres seraient en revanche métalinguistiques, tels les guillemets dont le signifié est lié à l'idée de locuteur ou de parole. Le statut sémiotique des signes de ponctuation est ainsi ambigu et complexe, en raison de la grande diversité des signes qui composent cet ensemble.

Face aux problématiques soulevées par l'usage du blanc, en poésie notamment, Henri Meschonnic propose de distinguer « ponctuation » et « signe de ponctuation » afin de pouvoir ressaisir, avec ce dernier terme, «l'ensemble des effets visuels d'une surface imprimée [...]»: «Ainsi le blanc n'est pas une absence de ponctuation, tout en étant une absence de signe de ponctuation $»^{45}$. Cette distinction féconde explique pourquoi nous avons fait le choix d'écarter, dans cette proposition, la ponctuation blanche («absence de signe »), cette dernière présentant des spécificités qui tendraient, à première vue - mais cela mériterait évidemment un traitement approfondi - à l'exclure de ce processus d'inclusion dans le système de la langue.

Si la question du signifiant graphique semble ne pas faire problème (Saussure lui-même ne fait pas de la dimension phonique un critère discriminant), la question du signifié, en revanche est centrale. Celle-ci semble conditionner l'assimilation définitive du signe de ponctuation noire au système de la langue. La question est donc de savoir s'il est possible d'établir un signifié réel, un concept, du côté de la signification en langue. Un courant contemporain, sous l'impulsion d'Yvan Fonagy et de Jacqueline Authier-Revuz, s'engage dans cette voie.

\subsection{Valeur en langue}

L'hypothèse de l'attribution d'un ensemble de traits définitoires afin d'établir une valeur distinctive des signes de ponctuation innerve la réflexion depuis de nombreuses années. Déjà la Grammaire Larousse, en 1936, évoquait la possible existence d'une " valeur précise »:

Sans doute chaque signe a une valeur précise, et ne peut être employé en dehors de cette signification : un minimum de ponctuation est nécessaire à la clarté d'un texte. 


\begin{abstract}
Mais chaque auteur a sa ponctuation personnelle plus ou moins fournie. Actuellement, dans des phrases généralement plus courtes, on ponctue plus qu'autrefois, en tenant compte du sens plus que de l'intonation. ${ }^{46}$
\end{abstract}

Apparaît ici, pour la première fois, le niveau de la langue, à travers la référence à la « valeur » et à la « signification » du signe. L'idée de l'attribution d'une valeur à chaque élément de ponctuation - puisqu'il n'existe pas, dans la ponctuation également, de synonymie absolue -, qui suppose d'établir un faisceau d'oppositions, d'aspects différentiels («toutes les valeurs sont d'opposition et ne se définissent que par leur différence $»^{47}$ ), émerge nettement. Derrière la structure logique se lit une fois de plus la tension entre les deux niveaux d'analyse avec, d'un côté, l'intuition d'une définition « sans doute » possible, a minima, du signe de ponctuation ("chaque signe a une valeur précise ») et, de l'autre, le constat d'une appropriation subjective du système par les auteurs (« mais chaque auteur a sa ponctuation personnelle »), qui vient ruiner l'invariance pressentie. L'aporie qui est ici présentée tient pour une part à la confusion des niveaux linguistique et discursif puisque, effectivement, un recensement des valeurs discursives relèverait très certainement de l'inépuisable.

Le premier à s'être intéressé, de façon approfondie, à la structure sémantique des signes de ponctuation est sans doute Ivan Fonagy ${ }^{48}$, pour qui il est possible de définir une fonction de base pour chaque signe. À partir de cette fonction de base (archifonction), l'analyse peut ensuite envisager les ramifications du réseau sémantique, à l'aune des différents éléments de l'énoncé affectés par l'intervention du ponctème. Cette conception, articulée autour de la fonction de base, des fonctions secondaires et de l'analyse des relations sémantiques, faisant se côtoyer sur un même plan signes linguistiques et signes de ponctuation, importe de fait les notions propres à l'analyse des unités lexicales (homonymie, synonymie et polysémie).

L'opposition formulée en premier lieu par Ivan Fonagy a été approfondie par Jacqueline Authier-Revuz qui établit la distinction entre valeur de base en langue et interprétations en discours. Étudiant les guillemets de modalité autonymique, Jacqueline Authier-Revuz postule qu'il existe, pour un tel signe, « une valeur, en langue, tout à fait définie, une, extrêmement abstraite et puissante » et qui peut se réduire à un «dédoublement opacifiant du dire d'un élément » ${ }^{49}$. Elle propose alors de faire le départ entre la valeur du signe en langue ${ }^{50}$ et le « continuum des valeurs interprétatives en discours de la valeur générale du signal » ou «l'espace interprétatif $»^{51}$ qui concerne l'ensemble des interprétations des signes en discours.

\footnotetext{
Ainsi, importe-t-il de distinguer ce qui est de l'ordre de la valeur du signal, constante, abstraite, réduite à la représentation opacifiante je dis $\mathrm{X}^{\prime}$ d'un élément du dire, et ce qui, suscité par cette valeur du signal, n'en est pas moins d'un autre ordre, celui du travail interprétatif, produisant, en discours, une variété de caractérisations non discrètes, non inventoriables, de X comme manière de dire : le guillemet de modalité autonymique n'est pas une marque renvoyant, de façon ambiguë, à un ensemble fini de valeurs - comme la réserve, la citation -, mais un signe non ambigu, à valeur générale - celle d'une pure opacification -, associé en discours à un ensemble non fini d'interprétations. ${ }^{52}$
}

Le départ entre « valeur » en langue et « ensemble non fini d'interprétations » en discours est adapté aux signes de ponctuation (la notion saussurienne de « valeur » qui rend compte du caractère différentiel du signe pourra être appréhendée à travers la signification - en langue - par opposition au sens - en discours). Les travaux récents sur la parenthèse (Pétillon) ou sur la note de bas de page (Lefebvre) témoignent de cette approche véritablement linguistique, en proposant une définition de l'élément ponctuant permettant d'envisager par la suite l'ensemble des réalisations discursives. Ainsi, la parenthèse est définie comme une « opération d'ajout » entraînant « la greffe d'un dire en plus $»^{53}$ et la note de bas de page, signe double fondé sur un « appel-renvoi de note», comme une « greffe typographique ${ }^{54}$. Les deux définitions procèdent selon une approche fonctionnelle (évoquant une « opération » ou un « appelrenvoi »), énonciative ou typographique. Il n'est pas encore question, véritablement, de valeur en langue. Ainsi, s'appuyant sur la même métaphore de la greffe dont la paternité peut être attribuée à Antoine Compagnon $^{55}$, elles présentent la particularité de mettre au premier plan la valeur de l'espace textuel plus que la valeur réelle du signe. Cette particularité est sans nul doute liée à la spécificité des signes doubles 
(ce qui inviterait alors à opérer une nouvelle distinction entre signes simples et signes doubles) et atteste de la multiplicité des approches possibles, en raison des particularités de chaque signe. Du fait de son caractère majoritairement diacritique, le signe de ponctuation ne s'extirpe pas aisément de son ancrage discursif. Jacqueline Authier-Revuz ne définit d'ailleurs pas les guillemets mais bien les guillemets de modalisation autonymique, ce qui suppose déjà de prendre appui sur une interprétation discursive.

S'il n'est pas encore établi que le signe de ponctuation puisse être doté d'une valeur et s'inscrire dans le système de la langue, ce nouveau questionnement et ces démarches linguistiques contemporaines traduisent néanmoins un changement d'appréhension, en s'engageant nettement dans la voie d'une étude à la fois énonciative et discursive, syntaxique et sémantique; la démarche qui vise à donner un statut aux signes dans le système de la langue, en le confrontant aux autres signes de ponctuation, indique bien, en elle-même, une évolution déterminante des conceptions, intéressant alors le processus de grammaticalisation.

\subsection{Grammaticalisation}

La morphogénèse de la ponctuation va dans le sens d'une prise en compte progressive des signes de ponctuation en tant qu'éléments grammaticaux. Ce mouvement de syntaxicalisation pourrait alors être apparenté à la notion de grammaticalisation, notion définie notamment par Christiane Marchello-Nizia :

Processus de changement dynamique, et unidirectionnel, par lequel des mots lexicaux ou des constructions syntaxiques changent de statut et acquièrent un statut de forme grammaticale. ${ }^{56}$

Il semble qu'on ne puisse envisager la place de la ponctuation dans le système de l'écrit sous cette acception, si l'on considère que le processus ne peut affecter que les "mots lexicaux» ou les «constructions syntaxiques». Toutefois, comme le rappelle Christiane Marchello-Nizia, le terme « grammaticalisation » recouvre deux sens. L'un désigne la description théorique du changement, c'est-àdire l'apparition des formes grammaticales dans les langues; l'autre envisage le résultat qui est la transformation de lexèmes en morphèmes ${ }^{57}$. L'évolution de l'appréhension de la ponctuation relèverait de la grammaticalisation au premier sens (apparition des formes grammaticales) mais, dans le détail, ne pourrait être reliée à la seconde acception, les signes de ponctuation n'étant évidemment pas des lexèmes. L'irréductibilité repose ainsi sur la nature des éléments soumis au processus. Or, en un sens plus large établi par Kurylowicz ${ }^{58}$, la grammaticalisation peut aussi concerner le cas d'un morphème se grammaticalisant davantage. Il serait alors possible, en laissant de côté la bipartition lexème/morphème, d'envisager la syntaxicalisation progressive de la ponctuation - à savoir une forme de " réanalyse ${ }^{59}$ telle que l'entend Bernard Combettes - comme un phénomène de grammaticalisation en étendant la définition restreinte de Marchello-Nizia à ce type particulier de morphèmes que seraient les signes de ponctuation.

Sur un plan diachronique, nous l'avons vu, le signe de ponctuation apparaît de plus en plus comme une unité grammaticale servant «à coder des informations qui n'étaient pas codées grammaticalement auparavant», ou «qui l'étaient mais différemment $»^{60}$. L'analyse de l'évolution des discours met en évidence un processus de changement dynamique tendant à une forme de codage, c'est-à-dire à l'inclusion des éléments ponctuants dans le système. Après Furetière, qui en était déjà à définir la ponctuation comme une « observation grammaticale », la syntaxicalisation progressive de la ponctuation observable à travers la description des grammairiens et des linguistes a pu contribuer à entériner une forme de grammaticalisation, dans la mesure où celle-ci « introduit des catégories qui n'avaient pas d'expression linguistique » et «transforme le système », selon la description de son fondateur, le linguiste Antoine Meillet ${ }^{61}$.

Reste que, dans ce processus global d'encodage grammatical, le questionnement contemporain sur la nature sémiotique du signe de ponctuation peut soulever quelques problèmes : dans le cadre d'une conception restreinte, unidirectionnelle, de la grammaticalisation, imposant l'idée d'un figement et d'une perte de sens, alors le fait d'envisager l'élément ponctuant comme un élément linguistique, doté d'un signifié et d'un signifiant (graphique), entrerait dans un processus inverse (de dégrammaticalisation ?). La 
grammaticalisation, au sens étroit et premier, induit en effet un processus d'érosion sémantique. Cependant, outre le fait que cette conception a depuis été nuancée, au profit d'une modification de sens, il est possible d'admettre que la volonté de poser un signifié en langue traduit un mécanisme de figement, désireux d'éliminer la polysémie en discours pour circonscrire le signe, pragmatiquement, dans une valeur unique: les changements de sens liés à la grammaticalisation peuvent être interprétés comme la conséquence d'une "pragmatisation ${ }^{62}$ croissante dans la langue. Ainsi, les démarches contemporaines s'intégreraient tout à fait dans ce mécanisme de changements linguistiques.

La notion de grammaticalisation - elle-même évolutive - présente de nombreuses variations, à l'inverse de la syntaxicalisation (sans doute moins polyvalente et moins ambigu), qui pour un cas aussi particulier que celui des signes ponctuation, peuvent faire obstacle à la clarté. Mais elle semble toutefois, à condition d'en accepter la mouvance et d'en définir précisément les enjeux, la plus à même de rendre compte d'un mouvement progressif et cohérent d'intégration des signes de ponctuation dans le système de la langue.

Bien entendu, une telle assimilation repose sur le caractère naturel de l'évolution, partant de la conception que l'on peut avoir de la langue. Le processus de grammaticalisation est une conséquence de l'activité du locuteur et non de la description théorique des spécialistes du langage. Il relève en quelque sorte davantage d'un sentiment linguistique, d'une perception, d'une intuition du scripteur sur l'essence des signes de ponctuation. Ce qui impliquerait d'arpenter un champ d'étude encore peu investi mais qui se donne comme « un poste d'observation privilégié $»^{63}$ : un imaginaire de la ponctuation. Un imaginaire ou encore un «sentiment » de la ponctuation, voire un "rêve » de ponctuation au sens où Gilles Philippe sonde, dans son dernier ouvrage, « le rêve d'un style parfait » ${ }^{64}$. S'il est malaisé de déterminer précisément dans quelle mesure la ponctuation est de plus en plus sentie par les locuteurs/scripteurs comme un élément grammatical bien plus que comme un élément pneumatique (du fait notamment qu'un tel sentiment puisse être en grande partie inconscient), il n'en demeure pas moins que ce glissement grammatical des discours autorisés peut apparaître comme le reflet d'une nouvelle appréhension intuitive des éléments de ponctuation.

\section{Conclusion}

Au terme de ce parcours, esquissé à grands traits, il apparaît que le champ de la ponctuation n'a cessé de s'accroître. En tendant vers le statut de signe linguistique, le signe de ponctuation peut s'emparer de tout ce qui compose l'analyse des unités lexicales. Certes, comme le souligne Isabelle Serça, « la question de savoir si la ponctuation relève au sens plein du système de la langue reste encore posée pour certains $»^{65}$. Le fait que la question reste posée n'empêche pas de considérer le questionnement en lui-même comme la conséquence d'un sentiment linguistique, devenu, au fil du temps, proprement grammatical.

Poussant la logique jusqu'au bout, on peut alors imaginer ce que serait une nouvelle (une ultime) étape de la morphogénèse des signes de ponctuation. Attribuer un signifié en langue au ponctème, c'est incontestablement rapprocher ce dernier de la notion de pictogramme, au détriment de celle d'idéogramme. En effet, ce qui fonde, en partie, la distinction entre idéogramme et pictogramme repose sur l'écart entre sens et signification : le sens de l'idéogramme, "signe mouvant ${ }^{66}$, évolue en fonction des multiples interprétations possibles en discours, quand le pictogramme, par-delà les variations, se signale par la permanence d'une signification primitive. Affirmer l'existence d'un signifié en langue contribuerait ainsi à faire du signe de ponctuation un pictogramme. Mais il faudrait, afin d'entériner cette transmutation, remettre également en cause ce qui constitue l'autre différence majeure entre les deux notions : le degré d'abstraction et le caractère arbitraire du signifiant graphique. Il s'agirait non seulement de reconnaître une forme d'iconicité du signe de ponctuation, mais aussi d'établir " des degrés dans l'arbitraire » du signifiant graphique, d'admettre que «le signe peut être relativement motivé ${ }^{67}$ ou de parvenir à identifier l'existence d'un élément de la valeur imposé du dehors par le jeu de l'analogie. Le signe de ponctuation ne serait plus seulement considéré comme un repère langagier mais comme une représentation graphique, schématisée, stylisée. Une nouvelle fois, cette évolution ne pourrait concerner au même degré tous les signes de ponctuation. Mais l'usage singulier, très contemporain, fait de certains 
signes dans le langage informatique/numérique - nous songeons ici, par exemple, à l'emploi autonome du point de suspension ${ }^{68}$ - invite à ne pas exclure complètement une telle perspective.

\section{Bibliographie}

Anis J. (1983). Pour une graphématique autonome. Le signifiant graphique, Langue française, $n^{\circ} 59$, p. 31-44.

Anis J. (2004). Les linguistes français et la ponctuation. L'Information grammaticale, $n^{\circ} 102$, p. 5-10.

Arrivé M. (2007). À la recherche de Ferdinand de Saussure. Paris : PUF.

Authier-Revuz J. (1995). Ces mots qui ne vont pas de soi. Boucles réflexives et non-coïncidences du dire, tome 2 Paris : Larousse, « Sciences du langage ».

Authier-Revuz J. (1998). Le guillemet, un signe de "langue écrite" à part entière. À qui appartient la ponctuation?, Paris, Bruxelles : Duculot, coll. « Champs linguistiques », p. 373-388.

Barko I. (1977). Contribution à l'étude de la ponctuation française au XVII ${ }^{\mathrm{e}}$ siècle (Problèmes de méthode - La ponctuation de Racine). La Ponctuation, recherches historiques et actuelles, tome 2, Paris et Besançon : CNRS et Groupement de recherches sur les textes modernes.

Benveniste E. (2001). Problèmes de linguistique générale, tomes 1 et 2, Paris : Gallimard.

Bikialo S., (2004). Le rivage de signes. Remarques sur la ponctuation et l'ailleurs. L'Information grammaticale, $n^{\circ} 102$, p. 24-30.

Catach N. (1977). La Ponctuation, recherches historiques et actuelles, tome 2, Paris et Besançon: CNRS et Groupement de recherches sur les textes modernes.

Catach N. (1982), «L'écriture et le signe plérémique », Modèles linguistiques, Tome VII, Fascicule 2, Presses Universitaires de Lille, p. 53-71.

Catach N. (1994), La Ponctuation, Paris : PUF, coll. « Que sais-je? ».

Catach N. (1998). La Ponctuation et les systèmes d'écriture : dedans ou dehors ? À qui appartient la ponctuation?, Paris, Bruxelles : Duculot. coll. « Champs linguistiques », p. 31-43.

Christin A.-M. (1995). L'Image ou la déraison graphique. Paris : Flammarion.

Combettes B. (2012). Réanalyse et discursivité. Sentiment de la langue et diachronie, Siouffi g. (dir.), Revue de linguistique française diachronique. Paris : PUPS, p. 132-152.

Compagnon A. (1979). La Seconde main ou le travail de la citation. Paris : Seuil.

Demonet M.-L. (2011). Ponctuation spontanée et ponctuation civile. La Ponctuation à la Renaissance, Paris : Classiques Garnier, p. 129-147.

Domergue F.-U. (1778). Grammaire françoise simplifiée, ou Traité d'orthographe, avec des notes sur la prononciation et la syntaxe, des observations critiques et un nouvel essai de prosodie, Lyon.

Drillon J. (1991). Traité de la ponctuation française. Paris : Gallimard.

Fonagy I. (1980). Structure sémantique des signes de ponctuation. Bulletin de la société de linguistique de Paris, $n^{\circ} 75$, p. $95-129$.

Furetière A. (1690). Dictionnaire Universel contenant généralement tous les mots français tant vieux que modernes et les termes de toues les sciences et les arts, tome 3, Genève : Slatkine Reprints.

Gaiffe F., Maille E., Breuil E., Jahan S., Wagner L., et Marijeon M. (avec la collaboration de) (1936). Grammaire Larousse du XXe siècle. Traité complet de la langue française. Paris : Larousse.

Gruaz N. (1980). Recherches historiques et actuelles sur la ponctuation. Langue française, $n^{\circ} 45$, p. 113-124.

Jaffré J.-P. (1998). Vers une linguistique de la ponctuation. À qui appartient la ponctuation ?. Paris, Bruxelles : Duculot, coll. « Champs linguistiques », p. 243-253.

Jenny L. (2012). Mises au point. «Et l'homme créa la page... ». Critique, $n^{\circ} 785$, p. 821-830. 
Lallot J. (1989). La Grammaire de Denys le Thrace. Paris : CNRS.

Lapacherie J.-G. (2000). De quoi les "signes de ponctuation" sont-ils les signes ?. La Ponctuation, La Licorne, $n^{\circ} 52$, p. 9-20.

Lefebvre J. (2004), "Note" et "note" : proposition de défrichage linguistique. L'Espace de la note, La Licorne, $n^{\circ} 67$, 2004, p. 27-50.

Lefebvre J. (2007). La note comme greffe typographique : étude linguistique et discursive. Thèse de doctorat nonpubliée, Sciences du langage, sous la direction de J. Authier-Revuz.

Lemare P.-A. (1819). Cours de langue française en six parties, tome 2, Paris.

Lignereux C., Piat J. (2009). "Tricher la langue ?". Une Langue à soi. Propositions. Bordeaux : Presses Universitaires de Bordeaux.

Marchello-Nizia Ch. (1978). Ponctuation et 'unités de lecture' dans les manuscrits médiévaux ou : je ponctue, tu lis, il théorise. Langue française, $n^{\circ} 40$, p. 32-44.

Marchello-Nizia Ch. (1979). Histoire de la langue française aux XIVe et XV siècles. Paris : Dunod.

Marchello-Nizia Ch. (2006). Grammaticalisation et changements linguistiques. Bruxelles : De Boeck..

Meigret L. (1550). Tretté de la grammere françoeze.

Meschonnic H. (2000). La ponctuation, graphie du temps et de la voix. La Ponctuation, La Licorne, n52, p. 289-293.

Pétillon-Boucheron S. (2002). Les Détours de la langue. Étude sur la parenthèse et le tiret double, Bruxelles : Peeters Louvain.

Perrot J. (1980). Ponctuation et fonctions linguistiques. Langue française, $n^{\circ} 45$, p. 67-76.

Phillipe G. (2013). Le Rêve d'un style parfait. Paris : PUF.

Rey-Debove J. (1977). Le Métalangage. Étude linguistique du discours sur le langage. Paris : Armand Colin.

Saussure F. (1976). Cours de linguistique générale. Paris : Payothèque.

Seguin J.-P. (1972). La Langue française au XVIII siècle. Paris : Bordas, Etudes.

Seguin P. (1993). L'Invention de la phrase au XVIII siècle. Bruxelles : Peeters publishers.

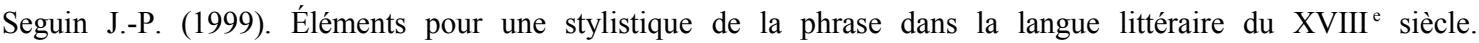
$L^{\prime}$ Information grammaticale, $n^{\circ} 82$, p. 5-15.

Serça I. (2004). La ponctuation : petit tour d'horizon. L'information grammaticale, $n^{\circ} 102$, p. 11-17.

Serça I. (2012). Esthétique de la ponctuation. Paris : Gallimard.

Sioufi G., Courbon B. (2012). Sentiment de la langue et diachronie, Revue de linguistique française diachronique, Paris : Presses de l'Université Paris-Sorbonne.

Tournier Cl. (1977). Pour une approche linguistique de la ponctuation. La Ponctuation : recherches historiques et actuelles, tome 2, Paris et Besançon : CNRS et Groupement de recherches sur les textes modernes.

Tournier Cl., (1980). Histoire des idées sur la ponctuation, des débuts de l'imprimerie jusqu'à nos jours. Langue française, $n^{\circ} 45$, p. $28-40$. 
${ }^{1}$ Voir par exemple Damourette J. (1939). Traité moderne de ponctuation, Paris : Larousse ; et très récemment, Doppagne A. (2006). La Bonne ponctuation. Clarté, efficacité et précision de l'écrit, Bruxelles: De Boeck. Duculot ou encore Eluerd R. (2002). Grammaire descriptive de la langue française, Paris : Armand Colin, coll. « Cursus ».

${ }^{2}$ Christin A.-M. (1995). L'Image ou la déraison graphique, Paris : Flammarion, coll. « Idées et Recherches ».

${ }^{3}$ Jenny L. (2012) Mises au point. "Et l'homme créa la page... », Critique, n785, tome LXVIII, p. 821. " Pratique impure » au sens où la ponctuation est tendue entre la fonction d'oralisation et celle d'identification et de structuration du texte écrit.

${ }^{4}$ Selon la distinction féconde opérée par Henri Meschonnic, que nous adoptons ici, le syntagme « signe de ponctuation » renvoie aux éléments de la ponctuation dite noire, par opposition au terme " ponctuation » qui inclut l'ensemble des éléments (La ponctuation, graphie du temps et de la voix. La Ponctuation, La Licorne, $n^{\circ} 52,2000$, p. 289-293, p. 290). Nous faisons le choix d'aborder, dans le cadre de cet article, uniquement les éléments idéogrammatiques de la ponctuation noire, laissant pour l'instant de côté la ponctuation blanche dont l'intégration dans le champ du signe pose des problèmes spécifiques (à commencer par la question du caractère discret).

${ }^{5} \mathrm{Au}$ sens où l'entend Benveniste, lorsqu'il oppose le sémiotique (la langue-système) au sémantique (le discours). Benveniste E. (1974). Sémiologie de la langue. Problèmes de linguistique générale, tome 2, Paris : Gallimard.

${ }^{6}$ Benveniste E. (1974). Problèmes de linguistique générale, tome 2, Paris : Gallimard/

${ }^{7}$ «La morphogénèse décrit le développement et la dynamique d'un phénomène, dans ses principes et dans ses formes les plus tangibles », J.-P. Jaffré. (1998). Vers une linguistique de la ponctuation. À qui appartient la ponctuation ?, Bruxelles : Duculot , coll. «Champs linguistiques », p. 243-253, p. 243.

${ }^{8}$ Serça I. (2012). Esthétique de la ponctuation, Paris : Gallimard, p. 40. Le système en trois points (point parfait - point d'en haut -, point médian et point d'en bas d'Aristophane de Byzance montre également, comme le révèle le commentaire de Denys le Thrace, la volonté d'atteler à la conception pneumatique (la respiration) une dimension logico-sémantique ("pensée complète », "pensée incomplète »). Il perdurera jusqu'au Moyen-Âge, avec une forte variation cependant en fonction des copistes. Denys le Thrace, élève d'Aristarque à Alexandrie, cité par Lallot J. (1989). La Grammaire de Denys le Thrace, Paris : CNRS, p. 92.

${ }^{9}$ Cité par Pocetti P. (2011). La réflexion autour de la ponctuation dans l'Antiquité gréco-latine. Ponctuation(s) et architecturation du discours à l'écrit, Langue française, $n^{\circ} 172$, p. 19-35, p. 33.

${ }^{10}$ Isidore de Séville, Etymologia, cité et traduit par P.-A. Lemare. (1819). Cours de langue française en six parties, tome 2, Paris : Bachelier, Huzard, p. 1212.

${ }^{11}$ Drillon J. (1991). Traité de la ponctuation française, Paris : Gallimard, p. 23.

${ }^{12}$ « On ne peut parler, à la limite, que de chaque manuscrit dans son unicité, et l'on ne sait rien de plus que ce que chaque manuscrit nous enseigne sur lui-même ", Marchello-Nizia Ch. (1978). Ponctuation et 'unités de lecture' dans les manuscrits médiévaux ou : je ponctue, tu lis, il théorise. Langue française, $n^{\circ} 40$, p. 44.

${ }^{13} \mathrm{Ibid}$.

${ }^{14}$ Catach N. (1998). La Ponctuation et les systèmes d'écriture : dedans ou dehors ? À qui appartient la ponctuation?, Paris Bruxelles : Duculot. coll. « Champs linguistiques », p. 31-43, p. 34.

${ }^{15}$ Serça I. (2004). La ponctuation : petit tour d'horizon. L'information grammaticale, $n^{\circ} 102$, p. 11-17, p. 12. Esthétique de la ponctuation, Paris : Gallimard, 2012, p. 40.

${ }^{16}$ Heynlin J. (1471). Compendiosus dialogus de arte punctuandi, cité par Marchello-Nizia Ch. (1979). Histoire de la langue française aux $X I V^{e}$ et $X V^{e}$ siècles, Paris : Dunod, p. 93.

${ }^{17}$ Furetière A. (1690). Dictionnaire Universel contenant généralement tous les mots français tant vieux que modernes et les termes de toues les sciences et les arts, tome 3, Genève : Slatkine Reprints, 1970.

${ }^{18}$ Beauzée N., Grammaire générale, cité par Gruaz N. (1980). Recherches historiques et actuelles sur la ponctuation. Langue française, $n^{\circ} 45$, p. $8-15$, p. 12

${ }^{19}$ Barko I. (1977). Contribution à l'étude de la ponctuation française au XVII ${ }^{\mathrm{e}}$ siècle (Problèmes de méthode - La ponctuation de Racine). La Ponctuation, recherches historiques et actuelles, tome 2, Paris et Besançon : CNRS et Groupement de recherches sur les textes modernes, p. 64

${ }^{20}$ Les éditions successives de 1694, 1762, 1798, 1832 du Dictionnaire de l'Académie française font apparaître le sens musical du terme (lié au silence) en second lieu, la première acception étant «l'intermission, la suspension, la cessation d'une action, d'un mouvement pour quelque temps » (Portail Atilf : http://portail.atilf.fr/cgi-bin/dicollook.pl?strippedhw=pause).

${ }^{21}$ Domergue F.-U. (1778). Grammaire françoise simplifiée, ou Traité d'orthographe, avec des notes sur la prononciation et la syntaxe, des observations critiques et un nouvel essai de prosodie, Lyon, p. 181.

${ }^{22}$ Demonet M.-L. (2011). Ponctuation spontanée et ponctuation civile. La Ponctuation à la Renaissance, Paris : Classiques Garnier, p. $129-147$, p. 135.

${ }^{23}$ Seguin P. (1993). L'Invention de la phrase au XVIII siècle, Bruxelles : Peeters publishers, coll. " Bibliothèque de l'Information Grammaticale », p. 13

${ }^{24}$ Seguin J.-P. (1999). Éléments pour une stylistique de la phrase dans la langue littéraire du XVIIIe siècle. L'Information grammaticale, $n^{\circ} 82$, p. $5-15$, p. 6. 
${ }^{25}$ « Combinée avec le recul de la période, l'étanchéité du point définitivement acquise forme le socle d'une stylistique de la phrase au XVIII' siècle », Ibid., p. 7

${ }^{26}$ Ibid., p. 8.

${ }^{27}$ Meigret L. (1550). Tretté de la grammere françoeze. http://gallica.bnf.fr/ark:/12148/bpt6k507854

${ }^{28}$ Lapacherie J.-G. (2000). De quoi les "signes de ponctuation" sont-ils les signes ? La Ponctuation, La Licorne, $n^{\circ} 52$, p. 9-20, p.14.

${ }^{29}$ Arrivé M. (2007). À la recherche de Ferdinand de Saussure, Paris : PUF, coll. « Formes sémiotiques », p. 150-151.

${ }^{30}$ Saussure F. (1916). Cours de linguistique générale, cité par Michel Arrivé, (2007), p. 150.

${ }^{31}$ Catach N. (1980). La ponctuation, La Ponctuation, Langue française, $n^{\circ} 45$, p. 27.

${ }^{32}$ Catach N., (1982). L'écriture et le signe plérémique. Modèles linguistiques, tome 7, fascicule 2, p. 53-71, p. 55.

${ }^{33}$ Tournier Cl. (1980). Histoire des idées sur la ponctuation, des débuts de l'imprimerie jusqu'à nos jours. Langue française, $n^{\circ} 45, \mathrm{p}$. 28-40, p. 36.

${ }^{34}$ « On note en effet que certains, comme la virgule peuvent permuter avec des mots grammaticaux. Ils sont donc comme des mots dans la phrase écrite. [...] Malgré les différences qui les opposent, en tant que signes, aux mots de la langue, on notera qu'ils présentent avec ceux-ci plusieurs traits communs. Ils sont susceptibles d'homonymie, de polysémie, de synonymie. ». Purnelle G. (1998). Théorie et typographie : une synthèse des règles typographiques de la ponctuation. A qui appartient la ponctuation ?, op. cit., p. 211-221, p. 213. La permutation évoquée ici implique une transformation nécessaire qui amène à relativiser les notions de " polysémie » et d' « homonymie ».

${ }^{35}$ "Notion confusionniste» pour Jacques Anis en raison de la confusion qui règne autour de l'emploi du terme « signe », Anis J. (2004). Les linguistes français et la ponctuation. L'Information grammaticale, $n^{\circ} 102$, p. 5-10, p.7.

${ }^{36}$ Tournier Cl. (1977). Pour une approche linguistique de la ponctuation. Ponctuation : recherches historiques et actuelles, tome 2 , Paris et Besançon : CNRS et Groupement de recherches sur les textes modernes, p. 252-270, p. 257.

${ }^{37}$ Tournier Cl. (1980). p. 36.

${ }^{38}$ Lapacherie J.-G. (2000). p. 15.

${ }^{39}$ Anis J. (1983). Pour une graphématique autonome. Le signifiant graphique, Langue française, $n^{\circ} 59$, p. 31-44, p. 41.

${ }^{40}$ Anis J., (2004). p. 7.

${ }^{41}$ Anis J., (1983). p. 41.

${ }^{42}$ Perrot J. (1980). Ponctuation et fonctions linguistiques. Langue française, $n^{\circ} 45$, p. 67-76, p. 67.

${ }^{43}$ Rey-Debove J. (1978). Le Métalangage. Étude linguistique du discours sur le langage. Paris : Armand Colin, coll. « U », p. 46.

${ }^{44}$ Ibid., p. 47.

${ }^{45}$ Meschonnic H. (2000). La ponctuation : graphie du temps et de la voix. La Licorne, op. cit., p. 289-290.

${ }^{46}$ Grammaire Larousse du XX siècle. Traité complet de la langue française. (1936). Avec la collaboration de Félix Gaiffe, Ernest Maille, Ernest Breuil, Simone Jahan, Léon Wagner, et Madeleine Marijon, Paris : Larousse, p. 127.

${ }^{47}$ Benveniste E. (1966). Problèmes de linguistique générale, tome 1, Paris : Gallimard, 2001, p. 54.

${ }^{48}$ Fonagy I. (1980). Structure sémantique des signes de ponctuation. Bulletin de la société de linguistique de Paris, $n^{\circ} 75$, p. 95-129.

${ }^{49}$ Authier-Revuz J. (1998). Le guillemet, un signe de "langue écrite" à part entière. À qui appartient la ponctuation ?, op. Cit., p. 373 388 , p. 380

${ }^{50} \mathrm{Ce}$ signifié de base est à bien distinguer de la forme schématique culiolienne. Avec le signifié en langue, il y a une valeur constante, une, qui apparaît dans l'énoncé, en filigrane. Cette notion insiste sur la permanence d'une valeur tandis que la notion de forme schématique s'appuie sur les variations et cherche à dégager une régularité derrière ces variations (un invariant). La notion d'invariant ne suppose pas l'existence d'un signifié en langue puisqu'elle interroge les régularités d'un phénomène de variations dans le discours pour en déduire un principe de changement dynamique (forme et déformations). La forme schématique suppose la prise en compte systématique du cotexte et envisage le processus d'interaction (elle informe le cotexte tout en étant déformée par les éléments du cotexte). Elle met l'accent sur les relations entre les unités dans le co-texte et semble être orientée davantage, du moins telle que nous l'entendons, dans le sens d'une approche sémasiologique quand le signifié en langue se donne comme une origine, un point de départ vers le discours et ses usages et apparaît plutôt du côté de la perspective onomasiologique : sa valeur est purement abstraite, relève de la langue et se saisit hors cotexte : c'est donc une forme d'abstraction d'une valeur minimale englobante et constante qui sera perceptible derrière chaque interprétations discursives.

${ }^{51}$ Authier-Revuz J. (1995). Ces mots qui ne vont pas de soi. Boucles réflexives et non-coïncidences du dire, tome 1, Paris : Larousse, coll. « Sciences du langage », p. 136-137.

${ }^{52}$ Ibid., p. 530-534.

${ }^{53}$ Pétillon-Boucheron S. (2002). Les Détours de la langue. Étude sur la parenthèse et le tiret double, Bruxelles : Peeters Louvain coll. « Bibliothèque de l'Information Grammaticale », p. 128. 
${ }^{54}$ Lefebvre J. (2007). La note comme greffe typographique : étude linguistique et discursive, Thèse de doctorat non-publiée, Sciences du langage, sous la direction de J. Authier-Revuz, p. 68.

${ }^{55}$ « La citation est un corps étranger dans mon texte, parce qu'elle ne m'appartient pas en propre, parce que je me l'approprie. Aussi son assimilation, de même que la greffe d'un organe, comporte-t-elle un risque de rejet contre lequel il faut me prémunir et dont l'évitement est l'occasion d'une jubilation. La greffe prend, l'opération réussit. [...] Le bâti doit disparaître sous la finition, et la cicatrice elle-même (les guillemets) sera un agrément supplémentaire ». Compagnon A. (1979). La Seconde main ou le travail de la citation, Paris : Seuil, p. 31-32.

${ }^{56}$ Marchello-Nizia Ch. (2006). Grammaticalisation et changements linguistiques, Bruxelles : De Boeck, p. 16.

${ }^{57}$ Ibid., p. 15.

${ }^{58}$ Cité par Christiane Marchello-Nizia, ibid., p. 18.

${ }^{59} \mathrm{La}$ notion de réanalyse est en effet beaucoup plus spécifiquement syntaxique, au sens qu'en donne par exemple Bernard Combettes lorsqu'il rappelle que cette opération consiste « pour le locuteur à assigner une nouvelle représentation syntaxique à une séquence de constituants déjà existants ", Combettes B. (2012). Réanalyse et discursivité. Sentiment de la langue et diachronie, Siouffi g. (dir.), Revue de linguistique française diachronique, Paris : PUPS, p. 131-152, p. 132.

${ }^{60}$ Marchello-Nizia Ch. (2006). p. 15

${ }^{61}$ Meillet A. (1912). Linguistique historique et linguistique générale, cité par Christiane Marcello-Nizia. (2006), p. 18.

${ }^{62}$ Hopper T., Traugott E.C. (1993). Grammaticalization, Cambridge : Cambridge University Press.

${ }^{63}$ Lignereux C. et Piat J. (2009). "Tricher la langue ?". Une Langue à soi. Propositions, Presses Universitaires de Bordeaux). Pour un développement éclairant sur la notion de "sentiment de la langue », voir également Sioufi G. et Courbon B. (2012). Sentiment de la langue et diachronie, Revue de linguistique française diachronique, Presses de l'Université Paris-Sorbonne.

${ }^{64}$ Philippe G. (2013). Le Rêve d'un style parfait, Paris : PUF.

${ }^{65}$ Serça I. (2004). p. 11.

${ }^{66}$ Christin A.-M. (1995). p. 47.

${ }^{67}$ Saussure F. (1972). p. 180.

${ }^{68}$ Le signe est ainsi répertorié dans diverses banques de données et d'archives de pictogrammes. Dans le langage informatisé, on le trouve en emploi autonome dans des onglets (rubrique inclassable, parfois accompagnée du sous-titre " autres ») mais aussi dans la communication textuelle afin d'indiquer au récepteur que le correspondant est en train d'écrire. 